\title{
Fire Sensor Data Logging
}

\author{
S. H. ELLWOOD \\ Gent Ltd. \\ Temple Road; Leicester, LE5 4JF, UK
}

\section{ABSTRACT}

Many fire alarm systems are being developed based on digital processing of signals from fire sensors. As background research for development of algorithms and sensors for such a system, a data logging exercise was undertaken using analogue fire sensors based on components from existing optical chamber smoke, Ionisation chamber smoke and heat detectors. The exercise was carried out using different areas within a factory which included offices, a laboratory, assembly shop, machine shop, warehouse and a kitchen. Data logging was also carried out on other sites which included a record of a small real fire incident in a hospital. Results are presented in the form of graphs of sensor output against time over time scales of approximately 1 week and 435 days. They are also presented as frequency plots of variations from a mean, on logarithm/1inear axes and, on natural logarithm against variation squared axes in order to test for a normal distribution. Sensor noise can be considered to be composed of several components, but that which causes most false alarms is dominated by signals which are artifacts of sensor and transmission faults. Algorithms should therefore be designed to reduce the effects of such faults, and also to diagnose incorrectly sited sensors.

Keywords

Fire detection, algorithms, fire sensors, data logging.

\section{INTRODUCTION}

The fire detection and alarm industry is currently undergoing widespread changes through the introduction of centralised processing of signals from analogue sensors by microprocessors. Many companies throughout the world are developing systems, and most of the emphasis is on the development of techniques for the transmission of signals and indicating the location of the fire signal. Many claims are made of these so-called intelligent systems the major one being reduction in false alarms. This is a very necessary aim if automatic fire detection is to gain more wide spread acceptance, as currently a fire alarm is just one of several ambiguous cues that people receive in a potentially dangerous situation (reference 1). 
There has not been much work reported on the algorithms to be used to distinguish real from false fire signals. Luck (reference 2) indicates an approach to this problem but indicates that there is also a lack of data in the normal behaviour of fire sensor signals which constitute the background noise. There have been several exercises on monitoring false alarms (reference 3 and 4), but little can be learnt from these about the behaviour of actual sensor signal levels. Burry is carrying out a research profect logging sensor signals and considering algorithms, but so far only preliminary results are available (reference 5). The work reported here is a similar exercise to that undertaken by Burry but done in paralle1 with the development of technology for the transmission and processing of signals for a distributed micro-processor fire alarm system. The system is now in commercial production and much of the information gained in this preliminary exercise has been incorporated.

Further work is in fact proceeding using the commercial equipment itself as a data-logger to determine algorithms to be used in more advanced versions of the product. This type of work has several aims:-

1. Gather data on back-ground noise for fire sensors.

2. Gather data on potential false alarms from fire sensors (which should be rejected).

3. Gather data on fires (which should be detected).

4. Gain knowledge of sensors and their signal properties which may lead to better designs of sensors and conventional detectors.

The data can then be used for statistical analysis and as input data to simulate processing in a fire alarm system. This paper will concentrate on the gathering of the data and the properties of the signals as observed from graph plots against time and by simple analyses.

\section{TECHNIQUE}

\section{Sensors}

The fire sensors used are all point type temperature or smoke sensors produced by utilising the sensor and mechanical parts of existing conventional detectors. These have been demonstrated to be amongst the most reliable in terms of fallure and false alarm rate in the industry. New electronic circuits were developed to produce analogue outputs of 0 to $5 \mathrm{~V}$ on the external contact pin normally used for a repeat indicating LED. Three types of point fire sensors were used in the work, the sensitivities being selected to give approximately equivalent outputs and responses.

A full series of fire tests (reference 6) were carried out to EN54 part 9 (reference 7) in order to establish the relative performance of the sensors with respect to different fire types.

Ionisation chamber smoke sensor. This is based on dual chamber design of sensor using a single $30,000 \mathrm{~Bq}$ Am241 source. It is similar in operation principle to many detectors in service but having the sensing gate of the impedance converting Field Effect Transistor connected to the source. The outer shell of the chamber is a mesh connected to 0 volts and the +12 volt supply is by a centre electrode spaced $1 \mathrm{~mm}$ from the source. 
The circuit was designed to give a unity gain transfer from the source voltage of the FET through to the output. The gajn of the sensors built was on average $1.46 \mathrm{~V} / \mathrm{y}$ with a standard deviation of $0.085 \mathrm{~V} / \mathrm{y}$ as measured in a smoke tunnel referenced to EN54 part 7 (reference 8). The alarm level. corresponds to a rise of $1.5 \mathrm{~V}$. This circuit has been designed to give variable d.c. offset to the FET source voltage to reduce the effect of the different quiescent output levels of each sensor. Each one was individually set to give a standing level of approximately 1.5V. In addition a semi-conductor temperature sensor on the printed circuit board was used to give a temperature compensation of $+10 \mathrm{mV} /{ }^{\circ} \mathrm{C}$ to correct for changes in sensor output with temperature.

Optical chamber smoke sensor. This senses smoke by scatter of infra-red radiation. The transmitter is a GaAs LED with a peak emission at $950 \mathrm{~nm}$ wavelength. The receiver is aligned at an angle of $50^{\circ}$ to the axts of the transmitter, and is a silicon photo-diode.

Pulses are generated in the circuit of $160 \mu$ s width at 6 times a second. The pulse is amplified through high gain circuit which is synchronised to the transmitter to reduce the effects of interference. The output is integrated to give a d.c. level.

The gain of the second amplifier stage was made adjustable and each one set to give a sensitivity of $10 \pm 2 \mathrm{~V} / \mathrm{dB} / \mathrm{m}$ as measured in a smoke tunnel to EN54 part 7 (infra-red obscuration). The alarm level corresponds to a rise of 1. $0 \mathrm{~V}$.

Temperature sensor. This uses most of the mechanics of a conventional heat detector but the bi-metalic element is replaced with a semi-conductor current source which acts as a sensor. The electronic circuit gives an output of:

$\mathrm{V}$ out $=1+0.04 \mathrm{~T}$.

Where $T$ is the sensor temperature in degrees centigrade. The alarm level corresponds to a rise to $3.4 \mathrm{~V}$.

\section{Data Logging Equipment and Software}

Two data loggers were used. They were commercial multiplex and A to D units connected to Hewlett Packard computers. The equipment was controlled by a programme written in BASIC.

The logger when running carried out the same condition for data storage as the commercial system uses for data transmission. This was convenient to reduce the data that was stored. Readings were taken at regular 1.25 second intervals. Every 256 th reading was stored but if a reading differed by that previously stored by more than a given threshold a series of 256 readings from that channel were also stored. This ensured that long term drifts and trends could be recorded along with high resolution data $j n$ regions of interest when the signal leve1s were changing rapidly. Each value was stored as a number in the range 0 to 255 corresponding to 0 to 5.12 volts, A set of 5 programs were written to log, transfer, and plot data. 


\section{Installation}

At each sensor location one of each of the ionisation, optical and temperature sensors were mounted on the ceiling along with a spare base. Multi-core screened cable was used throughout, a core being used for each sensor signal with a common supply and using the shield as a low jmpedance earth return.

One set of equipment was permanently installed at the Gent Factory in Leicester which gave a wide variety of room types and conditions. Twenty-two two locations were chosen which covered the following types of area:-

1. Development department office and laboratory.

2. Canteen and kitchen.

3. Warehouse and stores.

4. Works machine shop and electrical assembly areas.

5. Office, corridors and stairways.

A small set of equipment was used for logging on a series of sites remote from the factory, including fire tests and a period of data logging at $S t$ Crispins Hospital, Northampton.

\section{RESULTS AND DISCUSSIONS}

\section{St Crispins Hospital}

Nine continuous weeks were logged with sensors in a store room, bedroom, ward and day-room during the exercise. Most of the sensors showed very little variation above internal noise but figure 1 illustrates the reaction of the set in the day room. This was a psychiatric hospital and patients were allowed to smoke which may account for some of the variations in the ionisation sensor. Temperature was maintained very constant during day and night, the day-room in fact showing the greatest vartation seen.

The event at $1.256 \mathrm{E}+6$ seconds on the ionisation trace with a rise to above $3 \mathrm{~V}$ is of interest as it is a real fire caused by smoking material burning in a waste paper bin. The event in fact activated the fire alarm system installed in the hospital and even though was smal1 in size was considered a danger if staff were not present, due to the nature of the patients.

\section{Gent Factory}

observation of traces. The disk drive used had a capacity which required a change of disk each week to prevent the possibility of runing out of space. This section describes some of the observations made from traces plotted each week.

One effect which appears on traces of all types of sensor are random spikes. They are an artifact of the logging technique. Some (figure 2) are due to the transmission of voltage levels from fairly high impedance (10K Ohms) sources; they are generally worse during the working days and are due to electrical interference. These are not seen at $\mathrm{St}$ Crispins as there are less sources of interference and shorter cable runs. Others are a fault of the logging equipment and involve an occasional reading from an incorrect channel (figure 3 ). 


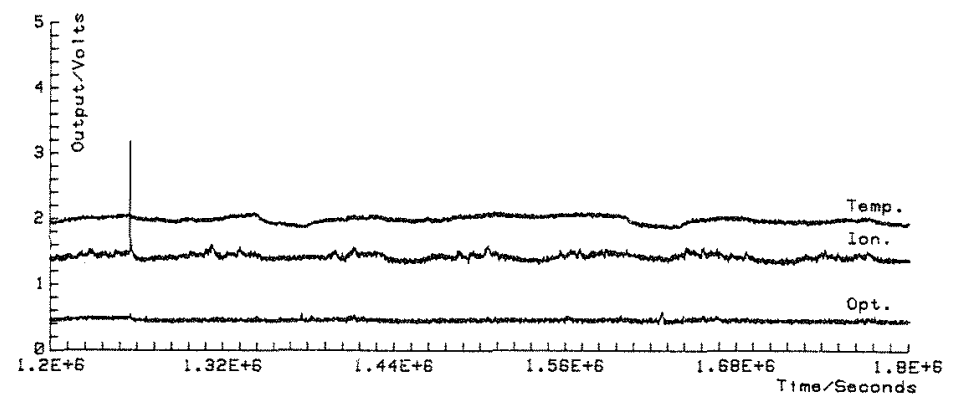

FIGURE 1. St Crispins Hospital, Day Room, fire at ionisation chamber smoke sensor.

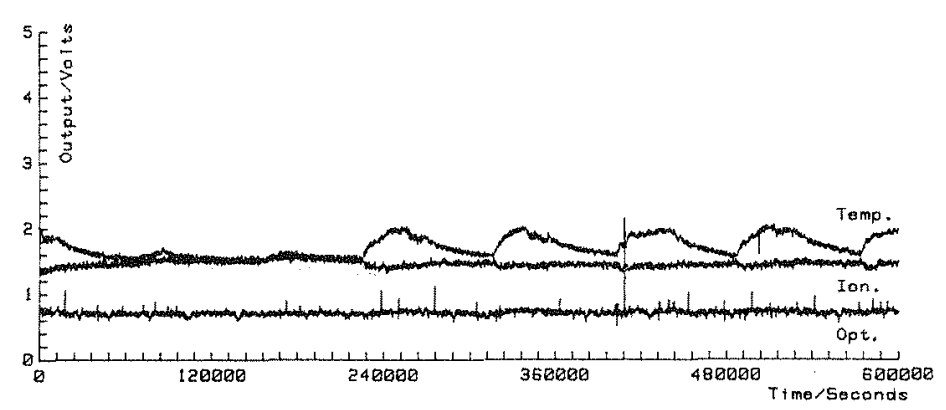

FIGURE 2, Gent Factory, Micro-lab.

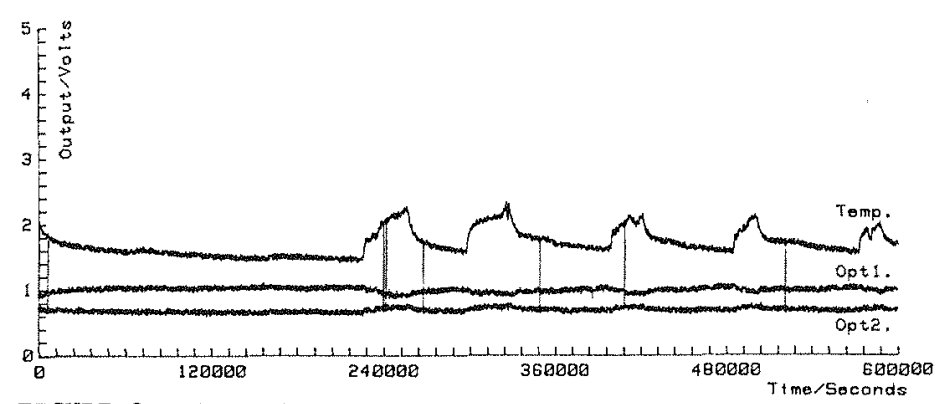

FIGURE 3. Gent Factory, Warehouse.

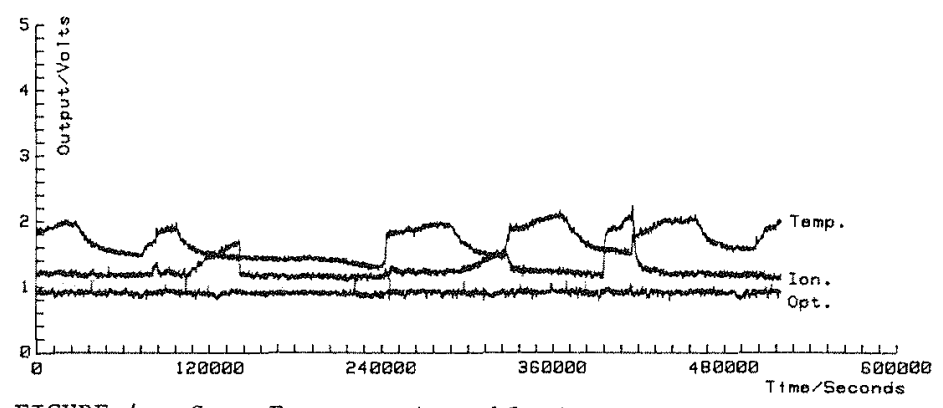

FIGURE 4. Gent Factory, Assembly Area. 
A fire detection system based on this logging technique would therefore be very unreliable; although it could be possible to filter out the effect of most of the spikes as they only affect single readings.

Temperature variations each day are much greater than at $\mathrm{St}$ Crispins and vary depending on the construction of the particular building being logged. The slow decay over the weekend after each change of disks on Fridays can clearly be seen on most traces as can the rise for each working day. Most of the sensors do not react to this daily change in temperature but some ionisation sensors exhibit a slight negative temperature coefficient (i.e. Figure 2), and optical sensors have been found which have both positive and negative temperature coefficients (Figure 3 ).

The stability of the heat sensors was the best of the three types with no failures, most of the noise being transmission faults on the line.

The ionisation sensors in general were stable but several failures were observed. This included the one shown in figure 4 which increased in signal as temperature decreased and humidity gradually increased during the night. The two small rises of approximately 0.5 and $0.3 \mathrm{~V}$ should have been sufficient to diagnose the sensor as faulty and prevent a false alarm in the event of later larger rises.

Unfortunately the optical smoke sensor was in the early stages of development when those used for the project were built, some were very noisy and had to be replaced, some had to be left in site and logged (Figure 2) whilst others behaved very well. This made apparent the need for very careful design of electronics of optical smoke sensors as the circuit has to have a very gain and be very stable to be reliable.

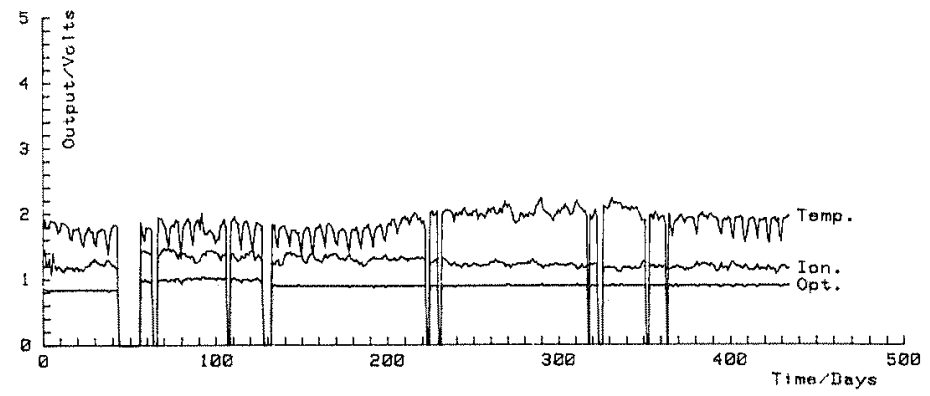

FIGURE 5. Gent Factory, Development office.

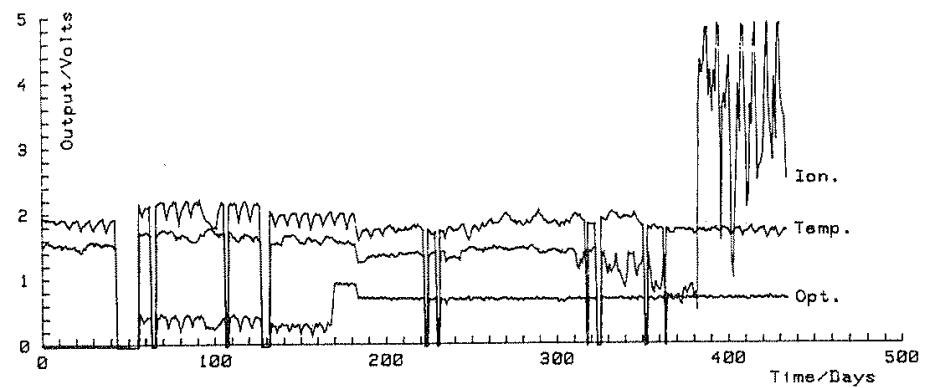

FIGURE 6. Gent Factory, Buying office. 
Very few signals were identifted which could be identified as due to smoke or other ajrborne particles which could not be explained such as regular temperature variations, noisy sensors or transmission faults. Some sensors that were placed in poor locations not normally suited to smoke detection did, however, exhibit some reaction. These included smoke sensors placed in a kitchen and a machine shop where smoke was sometimes present and some smoke sensors placed next to a heating unit.

Long term traces. After the projects had been running for over a year plots were compiled which give an impression of long term trends. The average over each day was calculated for each sensor logged and plotted. Effects not noticed in the short term can now be observed clearly. Periods when the logger was not operating was shown by a fall to oV.

The long term drift of $0.25 \mathrm{~V}$ of the ionisation smoke sensors can be observed (Figure 5) and is typical for all those used. This would create a reduction in sensitivity if a fixed threshold were used and demonstrates the need for cleaning and recalibration when conventional detectors are used. A system using a microcomputer can easily correct for this change. The weekly pattern of heating can be seen in the Winter and spring with a general rise in the summer. The optical trace shows step changes due to modification of the logging equipment.

Figure 6 illustrates a different location with the other ionisation sensor which was faulty. The failure can be observed to take place over several months with gradual decrease in stability and a falling level ending in a rise to high signal levels. With a conventional detector it would not be noticed until a false alarm occurred. The optical smoke sensor used at this location was changed after 170 days. Both optical sensors illustrated indicate the generally good long term stability observed for this type.

Veriations. Another form of overall impression can be observed from the frequency distribution of signal variations. To ensure that the distributions are not unnaturally welghted by the speed up of readings after a threshold, only the regular readings taken every 5 minutes were used. Three types of variation were compiled for each sensor:-

1. (Reading) - (average of 16 readings ( 1.4 hours)).

2. (Reading) - (average of 256 readings (22 hours)).

3. (Average of 16 readings ( 1.4 hours)) - (average of 256 readings (22 hours)).

As an example, traces from 8 ionisation sensors are shown (Figure 7 to 9). Two of those shown have given readings which could have caused an alarm, wh th positive variation greater than $1.5 \mathrm{~V}$ occurring during the exercise.

The variations in figure 7 are less than those in figure 8 , this is to be expected as they are with respect to a faster moving level. Those shown in figure 9 are generally very small and are plotted as indications of longer term drifts, probably mainly due to temperature fluctuation.

If the signals for the sensors are to be treated as random noise the probability of a signal deviation $x$ from a mean should be given by the normal distribution:-

$P(x)=\frac{1}{\sigma \sqrt{2 \pi}} \exp \left(\frac{-x^{2}}{2 \sigma^{2}}\right)$ 


$$
\text { or } \log _{e}(p(x))=\frac{-x^{2}}{2 \sigma^{2}}-\log _{e}\left(\frac{1}{\sigma \sqrt{2 \pi}}\right)
$$

from the data probability can be calculated by:-

$P(x)=\underline{\text { freq. }(x)}$

N

where freq. ( $x$ ) is the frequency of variations of $x$ and $N$ the total number of readings. Substituting in 3 gives:-

$$
\log _{e} \text { freq. }(x)=\frac{-x^{2}}{2 \sigma^{2}}+\log _{e}\left(\frac{N}{\sqrt{2 \pi}}\right)
$$

To test for a normal distribution a plot of $\log$ (freq. ( $x$ )) against $x^{2}$ should give a straight line of gradient $-1 / 2 e^{2}$. Figure 10 illustrates such a plot, each line represents the sum of all the sensors of each type used. The data for variations from the 22 hour average are plotted. None of the three curves are a straight line, but that for the ionisation sensor could be described by three straight segments:-

1. from the $y$ axis to point $A$, low levels of variation due to noise in the sensor, transmission and digitisation errors.

2. from point $A$ to point $B$, due to sensor noise, temperature variations, small fluctuations in air-bourne particle level. From the gradiente is $0.28 \mathrm{~V}$. This predicts a probability of a $1.5 \mathrm{~V}$ variation per year of $7 \mathrm{x}$ $10^{-3}$ for the 22 sensors used.

3. from point $B$ to the limit of the $x$ axis. This region is due to larger variations and can be identified as being mostly due to sensor faults and transmission errors.

The curve for the temperature sensor indicates a lower component of low level noise and a greater element of mid range variation. The optical result is dominated by sensor noise which is mainly due to the instability of the sensors used.

It is suggested that sensor noise in general consists of three components as indicated above. The third will normally be dominant as a cause of false alarms and as it consists mainly of artifacts of the sensor or data transmission system, it should be possible to engineer a system to decrease their effect. This should include algorithms which can identify sensors as faulty before they produced a fire-like signal or can reject signal shapes which need not bring up an alarm. A further cause of false alarms is that due to the presense of airborne particles at a high enough level to simulate a fire condition. These were rare in the exercise undertaken and it should be possible to minimise their effects by proper selection of sensor types or early identification of unsuitable areas by the system. 


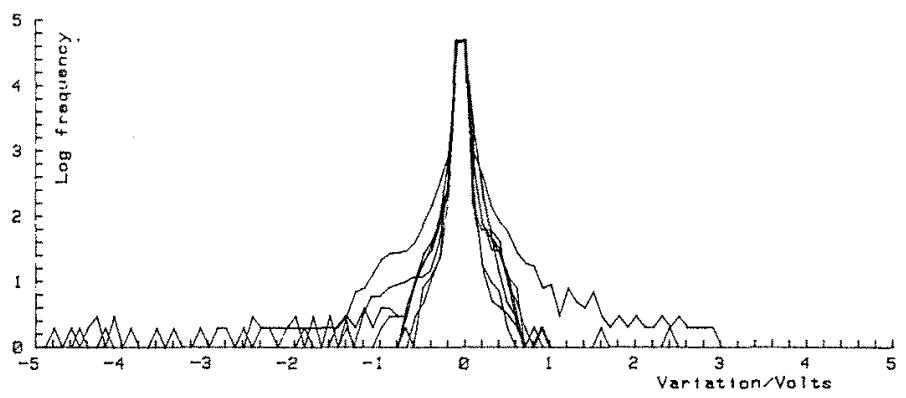

FIGURE 7. Gent Factory, 8 ionisation chamber smoke sensors, in warehouse, machine shop and assembly area, 5 min reading -1.4 hour average.

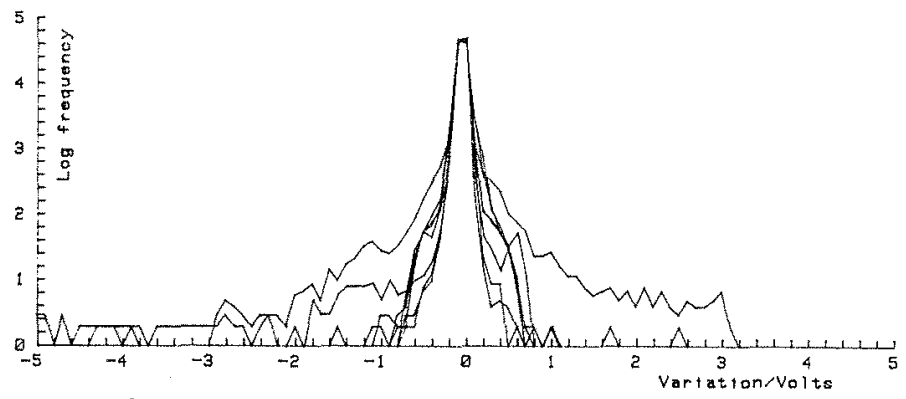

FIGURE 8. Gent Factory, 8 ionisation chamber smoke sensors, in warehouse, machine shop and assembly area, 5 min reading -22 hour average.

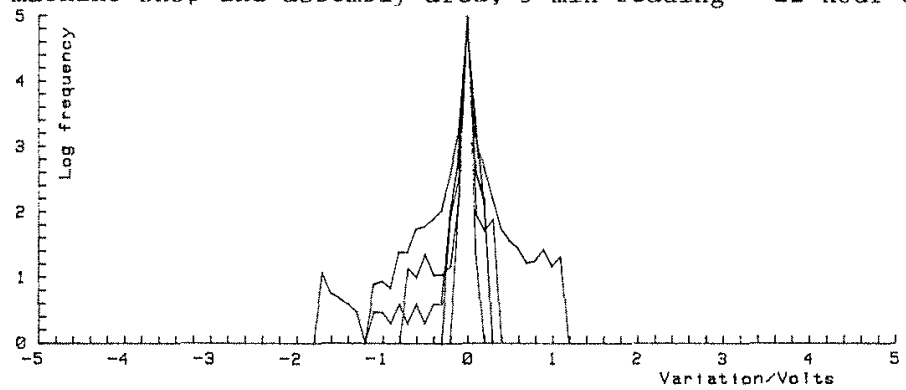

EIGURE 9. Gent Factory, 8 ionisation chamber smoke sensors, in warehouse, machine shop and assembly area, 1.4 hour average - 22 hour average.

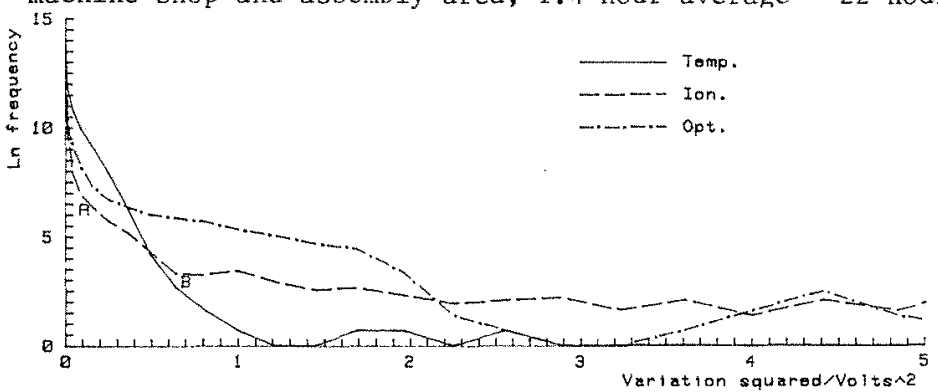

FIGURE 10. Gent Factory, All data logged fire sensors, 5 minute reading 22 hour average. 
Many results have been gathered as a result of this exercise (approximately one million sensor hours logged). Unfortunately the space only permits presentation of some of the more interesting events and observations. What has generally been of interest to observe is how sensors have failed. Most of the traces against time show little of interest as the vartance plots in the previous section demonstrates.

It has been interesting to note the different patterns that can be observed while looking at data over different timescales as shown by the different types of graph presented. The main aim of the work is to aid the development of algorithms for new generation fire alarm systems and as most of the effects seem to be artifacts of the system and the sensors it is clear that most effort needs to be directed in this area. As stated above false alarms would be rare if results were diagnosed early or sensors well sited. Both of these can be achieved. The data plotted indicates the type of signal that needs to be identified. Such an exercise needs to be repeated for each new design of sensor and transmission system, this will help to prove and develop the components and also provide information essential for the generation of algorithms and reliability data.

\section{REFERENCES}

1. Gantor, D., "Studies of human behaviour in fire: empirical results and their implications for education and design". B.R.E. report, BR61, 1985 .

2. Luck, H.O., "Correlation filters for fire detection systems", in Proceedings of International Symposium on Fire Safety Science, pp $749-758$, Hemisphere, 1986.

3. Gupta, Y.P., "Automatic fire detection systems: aspects of reliability, capability and selection criteria", Fire Safety Journal, 8, pp 105-117, $1984 / 85$.

4. Peacock, S.T., "Turnkey AFDS in the NHS data collection and analysis record report", University of Bradford report, 1986.

5. Burry, P., "False Alarms", B.R.E information sheet, 1986.

6. Ellwood, S.H., "Fire sensor data logging project", Gent Ltd report, 1985 .

7. BSI, EN54 part 9, BS5445 part 9, "Methods of test of sensitivity to fire" 1.984 .

8. BSI, EN54 part 7, BS5445 part 7, "Specification for point-type smoke detectors using scattered light, transmitted light or ionisation", 1984. 